\title{
A Neglected Case of Colonic Tuberculosis With Thoracolumbar Enterocutaneous Fecal Fistulae
}

\author{
Jie Tan ${ }^{\mathrm{a}}$, Daniel Porter ${ }^{\mathrm{b}}$, Jinxing Guo ${ }^{\mathrm{c}}$, Lijie Pan ${ }^{\mathrm{c}}$, \\ Guoshan Yang ${ }^{\mathrm{c}}$, Zichao Zhang ${ }^{\mathrm{c}, \mathrm{d}}$
}

\begin{abstract}
Although intestinal tuberculosis (ITB) typically affects ileocecal segments, the complication of tubercular enterocutaneous fistula is very rare. As an isolated phenomenon, primary intestinal manifestation without extraintestinal tuberculosis (TB) is exceptional and rarely reported. We present a patient with isolated ITB with six spontaneous thoracolumbar tubercular enterocutaneous fistulae. A 37-year-old Chinese woman was admitted to our institution complaining of escape of fecal matter through several openings in her back over the previous 20 years. She had nonspecific abdominal symptoms (occasional abdominal pain and alteration in bowel habit). External physical examination confirmed the presence of external thoracolumbar fecal fistulae in association with a scoliosis. Abdominal examination was unremarkable. Diagnostic colonoscopy with biopsy did not definitively confirm a diagnosis of TB. Microscopic examination in both microbiology and histopathology labs failed to identify acid-fast bacilli; however, gastrointestinal TB (GITB) was considered high on the differential diagnosis list. It was elected to perform open laparotomy with resection of the left hemi-colon. Postoperatively, she was treated with standard anti-tuberculous treatment for 6 months. The patient had an uneventful postoperative course, during which the fistulae gradually closed over the next year. This case with several low-output fistulas exiting the patient's back was successfully treated by a combination of surgery and antimicrobial therapy. The aim of this rare case report is to raise the awareness of atypical presentation of tubercular colonic enterocutaneous fistula so that timely diagnosis and intervention can salvage patient quality of life.
\end{abstract}

Keywords: Intestinal tuberculosis; Spontaneous enterocutaneous fis-

Manuscript submitted April 5, 2020, accepted April 9, 2020

${ }^{a}$ School of Clinical Medicine, Tsinghua University, Beijing 100084, China ${ }^{b}$ Department of Orthopaedics, The First Hospital of Tsinghua University (Beijing Huaxin Hospital), Beijing 100016, China

'Department of General Surgery, The First Hospital of Tsinghua University (Beijing Huaxin Hospital), Beijing 100016, China

${ }^{\mathrm{d}}$ Corresponding Author: Zichao Zhang, Department of General Surgery, The First Hospital of Tsinghua University (Beijing Huaxin Hospital), Beijing 100016, China. Email: zzc81518@163.com

doi: https://doi.org/10.14740/jmc3463 tula; Case report

\section{Introduction}

Intestinal tuberculosis (ITB) is very uncommon and typically affects ileocecal segments. Complications of ITB include bowel obstruction (31.7\%), intestinal perforation (4.9\%) and small bowel volvulus (2.4\%). Tubercular enterocutaneous fistula is very rare $(2.4 \%)$ [1]. There are very few case reports on ITB as a primary intestinal manifestation without extraintestinal tuberculosis (TB) $[2,3]$. We present a patient with a history of isolated ITB with neglected spontaneous enterocutaneous fistulae between the descending colon and the skin of the thoracolumbar region for more than 20 years.

\section{Case Report}

A 37-year-old female Chinese farmer with no significant past medical history was admitted to our institution in February 2012, complaining of escape of fecal matter through several openings in her back for the past 20 years. She first developed painful soft-tissue swellings under the skin of her back in 1992 at the age of 17. These pointed and discharged fecal matter mixed with pus after 4 weeks. Within a week, the opening healed, but then reappeared 2 weeks later, only to discharge again after a few days. This cycle of swelling followed by discharge continued until free-draining fistulae formed about 1 year later. Over the next 10 years, she developed an obvious thoracolumbar scoliosis. The patient was insistent that she had no spinal deformity prior to development of fistulae. Until 2012 the patient did not receive any treatment and her discharging fistulae continued to drain feces.

\section{Clinical findings}

Physical examination revealed a woman with appearances of chronic disease and an obvious thoracolumbar scoliosis. The abdomen was smooth and soft without peritonism or tenderness. Six fistulous $(0.5 \times 0.5 \mathrm{~cm})$ openings in the skin of her 

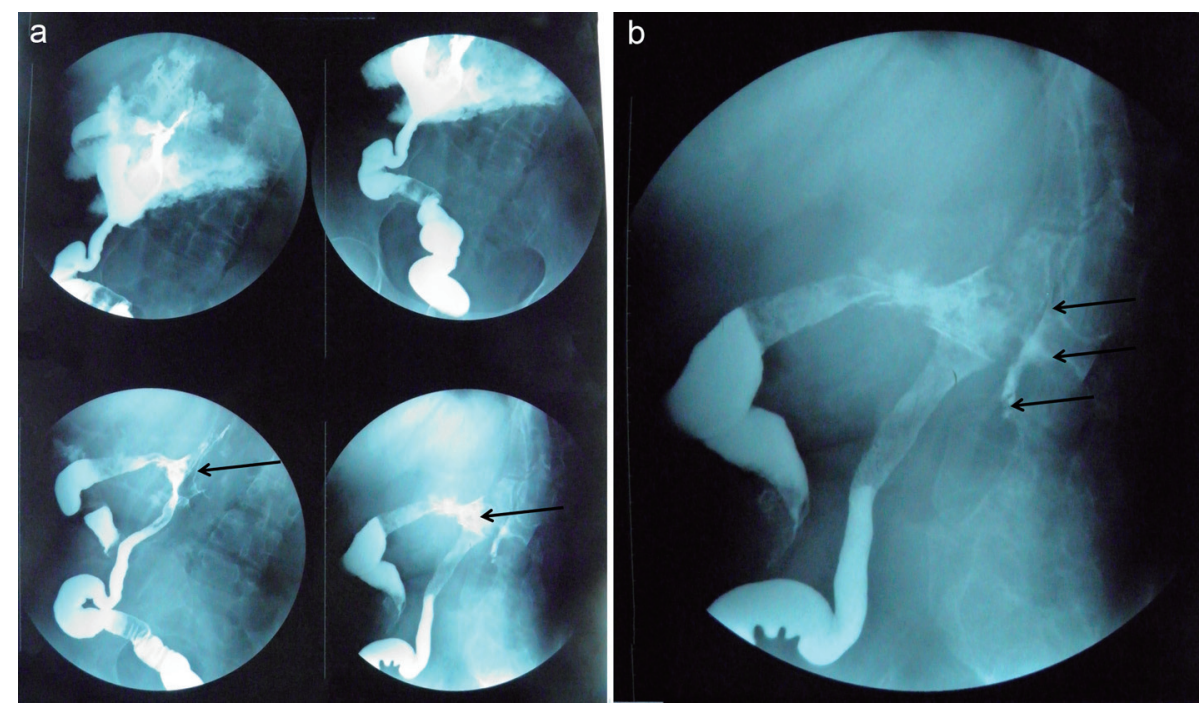

Figure 1. (a) Digestive tract barium studies showing fistulae (black arrows) between the colon and the thoracolumbar structures. (b) Fistulography showing multiple fistulae (black arrows) connecting the bowel lumen of the proximal descending colon to the exterior of the posterior abdominal wall.

back were identified with fecal soiling, surrounded by fibrous scar tissue.

\section{Diagnostic focus and assessment}

Chest X-ray did not show any manifestation of pulmonary TB. Abdominal CT revealed a fistulous track in the left posterior abdominal wall, mild splenomegaly and atrophic left kidney. Despite the scoliosis, vertebral bone quality appeared normal. Pelvic computed tomography (CT) showed sporadic lymphadenopathy ( $1-2 \mathrm{~cm}$ in size).

Digestive tract barium studies indicated fistulae between the colon and the thoracolumbar structures, with irregular stenotic segments of the rectum and sigmoid colon (Fig. 1a). Fistulography showed that there were multiple fistulae connecting the bowel lumen of the proximal descending colon to the exterior of the posterior abdominal wall (Fig. 1b).

Laboratory investigations revealed normal tumor markers, human immunodeficiency virus (HIV) test was negative, reverse passive hemagglutination reaction fecal occult blood test (RPHA-FOBT) was positive, white blood cells (WBCs) $3.31 \mathrm{Gpt} / \mathrm{L}$, NEUT\% 74.0\%, LYMPH\% 13.9\%, red blood cells (RBCs) $2.87 \mathrm{Tpt} / \mathrm{L}$, and erythrocyte sedimentation rate (ESR) $23 \mathrm{~mm} / \mathrm{h}$.

Subsequent colonoscopy confirmed colonic stenosis 30 - $35 \mathrm{~cm}$ from the anal margin. The colonic mucosa appeared slightly edematous with irregular hyperplasia, with no ulceration or tumorous lesion. Biopsies were taken from the abnormal mucosa and bowel wall. An opening $(\varphi=0.5 \mathrm{~cm})$ was seen at the apex of this edematous area, with back-flow confirmed when saline was injected into the external fistulous opening (Fig. 2). The mucosa of the rest of the colon was normal. Histologic analysis of biopsy specimens from the abnormal mucosa and bowel wall revealed non-specific inflammatory granu- lation tissue. There were no malignant cells or granulomatous tuberculous features. Acid-fast bacilli were not identified.

\section{Therapeutic focus and assessment}

Microscopic examination in both histology and microbiology laboratories had failed to identify acid-fast bacilli; however, since gastrointestinal TB (GITB) was considered high as a potential diagnosis laparoscopic surgery was performed. After extensive adhesiolysis, three sites of stenosis were identified with significantly thickened intestinal wall in the small bowel, approximately $10-20 \mathrm{~cm}$ proximal to the ileocecal valve. No small bowel resection was performed. Isolated swollen lymph nodes were found in the mesentery, one of which was sent for frozen section analysis. Intraoperatively, the descending and

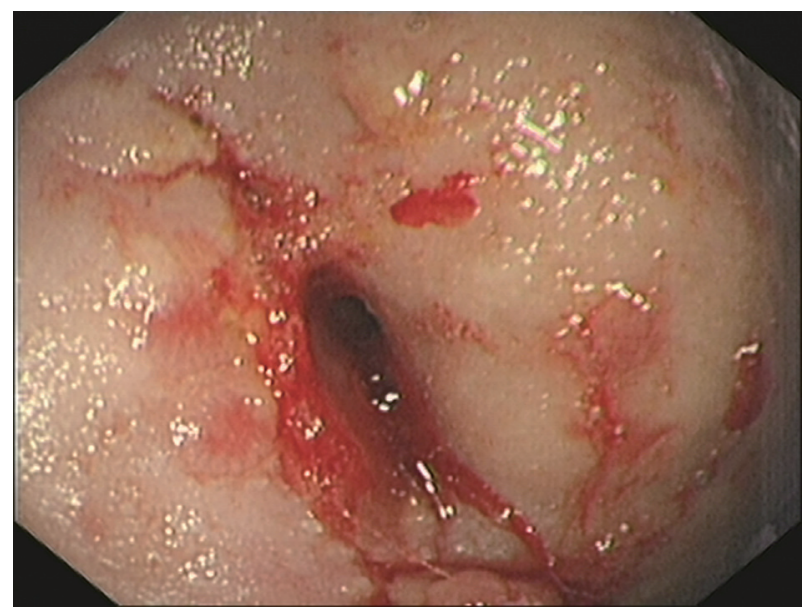

Figure 2. Colonic mucosa at colonoscopy: the fecal fistula aperture is clearly identified. 


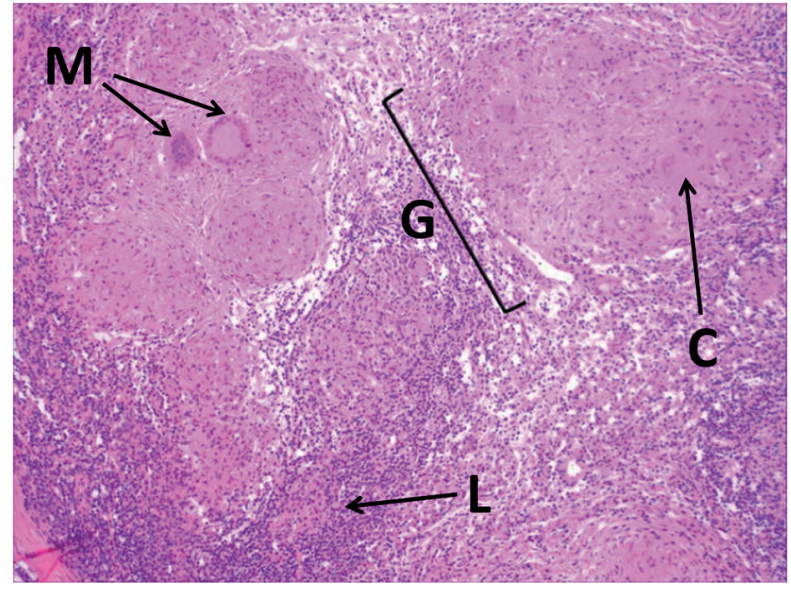

Figure 3. Photomicrograph of the intraoperative histopathology showing typical granulomatous features of tuberculosis: M: multinucleate giant cells; C: area of caseation within a granuloma; G: granuloma; L: lymphocytic infiltrate.

sigmoid colon was found to be densely adherent to the retroperitoneum surrounding a fistulous complex $(5.0 \times 4.2 \times 2.3$ $\mathrm{cm}$ ) extending through the soft tissues dorsally containing one internal and six external openings. Methylene blue injected by catheter confirmed a clear communication between colon and the exterior. Since the intraoperative frozen sections confirmed granulomatous inflammation, resection of the left colon was performed with primary reconstruction via a manual, single-layer, end-to-end anastomosis. The fistulous complex was carefully dissected from surrounding normal tissue and excised en bloc with abnormal bowel.

\section{Follow-up and outcomes}

The patient had an uneventful postoperative course. Definitive histopathological examination of the resected specimen confirmed the presence of caseating granulomas (Fig. 3).

She was commenced on standard anti-tuberculous quadruple therapy. The external fistulae gradually closed entirely over the following year. The patient returned for follow-up over 4 years. At final clinical review she presented in very good general condition: free of any symptoms related to colonic TB.

\section{Discussion}

TB remains endemic in developing countries worldwide, largely as a result of the epidemic of acquired immune deficiency syndrome (AIDS), multidrug-resistant TB and greater levels of poverty and migration [4]. Pulmonary disease is the most common manifestation of TB. Fewer than $5 \%$ of patients have extrapulmonary manifestations and only $1 \%$ of patients have the gastrointestinal tract affected [5]. Furthermore, only a minority of patients with TB who are not infected with HIV have gastrointestinal involvement $[6,7]$.
ITB is relatively uncommon, and typically affects ileocecal segments. It is usually found in association with extraintestinal TB infection. The infection sources include swallowed infected sputum, hematogenous or contiguous spread from adjacent organs or contaminated food [8]. More commonly, intestinal inflammation with ulcers, strictures, and hypertrophic lesions such as polyps or masses mimicking a Crohns-like or cancer-like disease are common presentations $[9,10]$. Symptoms range from only mild, non-specific abdominal pain to enteritis with diarrhea or intestinal obstruction and even perforation. Compared with primary abdominal TB spontaneous tubercular enterocutaneous fistula is very rare [11]. Moreover, a primary intestinal manifestation without extraintestinal TB is exceptional and rarely reported $[2,3,9]$. We present a patient with isolated GITB with spontaneous tubercular enterocutaneous fistulae developed in the back. She had neither HIV infection nor extraintestinal TB, and no significant previous past medical history.

Preoperative confirmatory histology of underlying TB is often difficult in the abdomen, especially in the absence of radiological evidence of pulmonary disease [12]. Accurate imaging of the fistula track can be gained by fistulography and barium studies. Furthermore, in a significant proportion of patients (20-40\%), histologic analysis of mucosal biopsies shows only chronic, nonspecific, inflammatory changes, leading to no definitive diagnosis [13]. In this case also, histologic analysis of colonoscopic biopsy specimens from the abnormal mucosa and bowel wall revealed many inflammatory cells within a matrix of granulation tissue.

TB is one of the main underlying pathologies for enterocutaneous fistulas and the small intestine is the most common site of origin [14]. Measurement of the output of the fistula and classification of low- and high-output varieties has implications for management [15]. For high-output fistulas, enteric fever is the most common cause $(45 \%)$ and the ileum is the most likely site of origin (82\%) [14]. Most high-output fistulae are associated with systemic sepsis (63\%), and mortality is over $50 \%$ [14]. High-output fistulae usually require surgical intervention, while low-output fistulae in well-preserved individuals may be managed conservatively [15]. In this case, there were several low-output colonic fistulae exiting in the skin of the back with no sign of sepsis. However, resection of all the fistulae tracks in their entirety at the same time was considered unwise due to the large resultant skin defect. Instead we elected to excise the fistulous complex with the longest possible fistula track included and the internal opening of the fistula was electrocauterized followed by the standard tuberculostatic treatment. The patient had an uneventful smooth postoperative course and the external opening gradually healed over the next year after surgery.

The obvious thoracolumbar scoliosis (which the patient insisted developed 10 years after the onset of fistulae) requires comment. There were no prior radiographs available and the patient came from a very poor family which did not seek any medical attention for her until recently. The normal vertebral bone quality on X-ray and CT suggests that there was no direct spinal involvement in the tuberculous process. It is interesting to speculate whether the abnormal local pathological process might have had a role in initiation of spinal curvature, albeit after skeletal maturity. Scoliosis may have been initiated by the 
abnormal forces created by the pathological process in close proximity, possibly through chronic muscle spasm or fibrosis; however, this degree of curvature forming de novo in a skeletally mature individual must be extremely rare. We could find no similar examples in the medical literature.

\section{Conclusions}

This case with several low-output fistulae in the thoracolumbar region was treated successfully by a combination of judicious surgery and antimicrobial therapy. The aim of this rare case report is to raise the awareness of atypical presentation of tubercular colonic enterocutaneous fistula so that timely diagnosis and intervention can salvage patient quality of life.

\section{Acknowledgments}

None to declare.

\section{Financial Disclosure}

None to declare.

\section{Conflict of Interest}

None to declare.

\section{Informed Consent}

The patient agreed to provide her information for the publication of this case report.

\section{Author Contributions}

JT and ZZ participated in the literature search and drafted the manuscript. JG, LP and GY participated in the notes review. $\mathrm{ZZ}$ and DP conceived the study and participated in its design and coordination and helped to draft the manuscript. All authors read and approved the final manuscript.

\section{Data Availability}

The data supporting the findings of this study are available from the corresponding author upon reasonable request.

\section{References}

1. Ceccherini E, Sereni P, Felicioni L, Testi W, Mancini S. [Tubercular enteral fistulas]. Minerva Chir. 1989;44(8):1293-1295.

2. Conzelmann M, Zenklusen HR, Fried R, Frei R, John H, Huber F. [Unusual intestinal manifestations of tuberculosis]. Schweiz Med Wochenschr. 1993;123(6):234-239.

3. Gupta NM, Motup T, Joshi K. Isolated colonic tuberculous perforation as a rare cause of peritonitis: report of a case. Surg Today. 1999;29(3):273-275.

4. Raviglione MC, Snider DE, Jr., Kochi A. Global epidemiology of tuberculosis. Morbidity and mortality of a worldwide epidemic. JAMA. 1995;273(3):220-226.

5. Sbarbaro JA. Tuberculosis in the 1990s. Epidemiology and therapeutic challenge. Chest. 1995;108(2 Suppl):58S$62 \mathrm{~S}$.

6. Khan R, Abid S, Jafri W, Abbas Z, Hameed K, Ahmad $Z$. Diagnostic dilemma of abdominal tuberculosis in non-HIV patients: an ongoing challenge for physicians. World J Gastroenterol. 2006;12(39):6371-6375.

7. Epstein D, Watermeyer G, Kirsch R. Review article: the diagnosis and management of Crohn's disease in populations with high-risk rates for tuberculosis. Aliment Pharmacol Ther. 2007;25(12):1373-1388.

8. Horvath KD, Whelan RL. Intestinal tuberculosis: return of an old disease. Am J Gastroenterol. 1998;93(5):692696.

9. Misra SP, Misra V, Dwivedi M, Gupta SC. Colonic tuberculosis: clinical features, endoscopic appearance and management. J Gastroenterol Hepatol. 1999;14(7):723729.

10. Pulimood AB, Ramakrishna BS, Kurian G, Peter S, Patra S, Mathan VI, Mathan MM. Endoscopic mucosal biopsies are useful in distinguishing granulomatous colitis due to Crohn's disease from tuberculosis. Gut. 1999;45(4):537541.

11. Rao PL, Mitra SK, Pathak IC. Spontaneous tuberculous enteroumbilical fistulas. Am J Gastroenterol. 1979;72(6):671675.

12. Singh O, Gupta S, Moses S, Jain DK. Spontaneous tubercular enterocutaneous fistula developing in the scar of a surgery done 14 years earlier. Saudi J Gastroenterol. 2009;15(4):261-263.

13. Shah S, Thomas V, Mathan M, Chacko A, Chandy G, Ramakrishna BS, Rolston DD. Colonoscopic study of 50 patients with colonic tuberculosis. Gut. 1992;33(3):347351.

14. Kaur N, Minocha VR. Review of a hospital experience of enterocutaneous fistula. Trop Gastroenterol. 2000;21(4):197-200.

15. Leslie MD, Slater ND, Smallwood CJ. Small bowel fistula from a Littre's hernia. Br J Surg. 1983;70(4):244. 